Erratum

\title{
Extremes of nonexchangeability
}

Roger B. Nelsen

Department of Mathematical Sciences, Lewis \& Clark College, Portland, Oregon 97219, USA

Erratum to: Statistical Papers 48: 329 - 336 (2007)

Due to an unfortunate error, the name of the author was not correct. 